\title{
QUALITÄTSSICHERUNG UND \\ DEREN ENTWICKLUNG AUS SICHT \\ DER FH OBERÖSTER-REICH \\ EIN RESÜMIERENDER BLICK AUF DAS GESTERN UND HEUTE - MIT EINER ÜBERLEGUNG FÜR MORGEN...
}

REGINA AICHINGER

DOI: $10.22163 /$ fteval.2019.451

\author{
»WER DIE VERGANGENHEIT NICHT \\ KENNT, KANN DIE GEGENWART \\ NICHT VERSTEHEN UND DIE \\ ZUKUNFT NICHT GESTALTEN.॥
}

Helmut Kohl 1995

\section{QUALITÄTSSICHERUNG IM ÖSTERREICHISCHEN FH-SEKTOR}

$\mathrm{M}$ it der Gründung des österreichischen Fachhochschul(FH)Sektors durch Beschlussfassung des FH-Studiengesetzes (FHStG) im Jahre 1993 wurde ein Fundament geschaffen, das innovative legistische Determinationen in Richtung bildungsstrategisch und -ökonomischer Ziele setzte. Das Leistungsportfolio für fachhochschulische Einrichtungen wurde durch kompetitive Mitteleinwerbung für Studienplatzfinanzierung sowie konsequente Leistungsorientierung aufgrund einer Kombination aus interner und externer Qualitätssicherung (OS) nachhaltig geprägt. ${ }^{1}$ Vielfach wurden/werden hierzu etablierte Qualitätsmanagement(QM)-Modelle und -Methoden eingesetzt. Mit diesen Rahmenbedingungen finden sich die Fachhochschulen in ein qualitätsgeleitetes Governance-Konzept eingebunden, das auf Systemebene wesentliche Berichts- und Nachweispflichten gegenüber dem zuständigen Bundesministerium für Bildung, Wissenschaft und Forschung, der Agentur für Qualitätssicherung und -akkreditierung Austria sowie, auf institutioneller Ebene, den Eigentümern und darüber hinaus der Öffentlichkeit gegenüber vorsieht. Diese dienen einer möglichst erfolgreichen Positionierung innerhalb eines kompetitiven Umfeldes: Und hier wiederum im Speziellen zur Legitimierung aufgrund erfolgreicher Akkreditierung von FH-Studiengängen ${ }^{2}$ sowie der gegenwärtig alle sieben Jahre zu erfolgenden Zertifizierung des hochschuleigenen OMSystems.

\section{EXTERNE QUALITÄTS- SICHERUNGSVERFAHREN UND DEREN ENTWICKLUNG ${ }^{3}$}

Im Hinblick auf die externe Qualitätssicherung ist im Zeitverlauf eine kontinuierliche Weiterentwicklung in begrifflicher wie inhaltlicher Hinsicht zu beobachten. In den Jahren 1993-2002 dominierten im Bereich der externen, behördlichen Qualitätssicherung zunächst studiengangsbezogene Überprüfungen. Die ersten österreichweit durchgeführten Evaluierungen von 10 FH-Studiengängen wurden noch unter Anwendung der Methodik des „problemzentrierten Interviews" abgewickelt: Ziel war, „[...] die evaluierten Prüfbereiche, ihre Ursachen und Lösungswege [...]" zwischen Vertretern des damals als Bundesbehörde zuständigen Fachhochschulrates (FHR) sowie der FH-Erhalter zu besprechen und damit schrittweise ein (fach-)hochschulisches Qualitätsbewusstsein zu entwickeln. ${ }^{4}$ Der zu diesem Zeitpunkt eingesetzte Mix aus formativer wie summativer Evaluierung (Akkreditierung und Re-Akkreditierung) sollte sicherstellen, dass Abweichungen zwischen definierten Soll- zu identifizierten Ist-Zuständen sichtbar gemacht sowie

Das damit an den Fachhochschulen etablierte Qualitätsmanagement hat sich an den gesetzlichen Bestimmungen des Hochschul-Qualitätssicherungsgesetzes (HS-OSG), dem Fachhochschul-Studiengesetz (FHStG) sowie den einschlägigen Verordnungen der Agentur für Qualitätssicherung und -akkreditierung Austria (AQ Austria) zu orientieren.

2 Vgl. Fachhochschul-Entwicklungs- und Finanzierungsplan, FHStG BGBI. 390/1993 §8 idgF, HS-OSG BGBI. I 74/2011 §22 idgF.

3 Die gegenständliche Betrachtung umfasst den Begriff der Qualitätssicherung in erster Linie mit Bezug auf die behördlichen Qualitätsverfahren und nicht die automatisiert zu erbringenden Leistungsnachweise bzw. Datenmeldungen.

$4 \quad$ Vgl. Kozar, G, 1999: Hochschul-Evaluierung, in: Schriftenreihe des FHR, WUV-Verlag, Wien, S. 67. 
Maßnahmen zur Qualitätssteigerung geplant und umgesetzt wurden. Die rasche Entwicklung des Sektors (10 FH-Studiengänge und 7 Erhalter im Studienjahr 1994/95, 124 Studiengänge und 17 Erhalter im Studienjahr 2002/03) erforderte schließlich eine Veränderung der geübten Praxis. Mit 2003 wurde zusätzlich die institutionelle Evaluierung ${ }^{5}$ als eigenes Verfahren eingeführt, um die FH-Einrichtungen nach internationalen Standards ${ }^{6}$ dazu anzuleiten, organisationale Verantwortung zu entwickeln. Die institutionelle Evaluierung sollte vor allem eine Abkehr von punktueller, externer Leistungsnachweiskontrolle der Studiengänge darstellen und die FH-Einrichtungen dazu anleiten, ganzheitliche OM-Systeme zu etablieren.? Damit war die Erwartung verbunden, Qualitätsarbeit nicht nur als zeitlich determinierte „Fehlerbehebungsroutine" zu betreiben, sondern vielmehr Qualitätsbewusstsein in den Hochschulalltag zu integrieren. Für die FH-Einrichtungen war damit vor allem der Fokus auf die Etablierung einer integrativen, strukturellen und prozeduralen Rahmensetzung in der Qualitätsarbeit wesentlich. Eine weitere Änderung erfuhr die externe Qualitätssicherung im Jahr 2006: Hier wurde die Koordination der Evaluierungen aus dem FHR ausgelagert und die FH-Einrichtungen dazu angehalten, sich zur Verfahrensabwicklung einer anerkannten Qualitätssicherungsagentur zu bedienen. Weiters wurde zu diesem Zeitpunkt inhaltlich die externe Qualitätssicherung mit einem Verfahren und nach der Logik des „informed peer-review” eingeführt, wobei dieses eine Selbstanalyse und -bewertung der fachhochschulischen Einrichtung sowie eine spiegelgleiche Bewertung seitens einer Expert*innengruppe von externer Seite vorsieht. Eine Verfahrenslogik, die sich bis dato erhalten hat bzw. Anwendung findet. Die vorläufig letzte größere Änderung in der externen Qualitätssicherungslogik fand 2012 mit der Beschlussfassung des HS-OSG und der Novelle des FHStG zu diesem Themenbereich statt. Die studiengangsbezogenen Re-Akkreditierungen wurden aufgegeben, nur noch Erst-Akkreditierungsverfahren definiert und ergänzend die institutionelle Evaluierung terminologisch durch den Begriff des „institutionellen Audits"8 ersetzt. Die inhaltlichen Stoßrichtungen und Kriterien von bzw. für Akkreditierungen und Audits setzen laufend neue Maßstäbe.

\section{DAS QUALITÄTS- MANAGEMENTSYSTEM DER FH OBERÖSTERREICH}

Gemäß einer Grundidee der European Standards and Guidelines for Quality Assurance in Higher Education (ESG) umfasst die hochschulische Qualitätssicherung, " [...] alle Aktivitäten im Rahmen des kontinuierlichen Verbesserungsprozesses (dh. Sicherung und Verbesserung der Qualität) [... $]^{\prime \prime} .{ }^{9}$ Eine fachhochschulische Einrichtung, die den Anspruch stellt, aus der Geschichte zu lernen und Prozesse wie Strukturen zu etablieren, um leistungsfähig zu sein und zu bleiben, stellt sich die Frage, wie die skizzierte Entwicklung der Qualitätssicherung zu bewerten ist. In der Diskussion um externe Hochschul-Qualitätssicherung könnte eine organisationspädagogische Betrachtung angestellt werden: Hier wird nach dem Grundsatz der „[... motivationalen Steuerung des Verhaltens [...]" ein Subjekt (Individuum/Organisation) dann als lern- und veränderungsbereit betrachtet, wenn ein intrinsisch motiviertes Bestreben nach Kompetenzauf- und -ausbau existiert. Dann wird eine externe Anreizsetzung zum Lernen - hier mit dem Ziel möglichst hoher qualitativer Performance - ermöglicht. Allerdings auch mit der Maßgabe, dass die Weiterentwicklung selbstbestimmt erfolgen kann, die Leistung mit Legitimierung und Reputationswahrnehmung bzw. Anerkennung einhergeht und ein Vertrauensbezug und Zugehörigkeitsempfinden zu einer Lehr-/Lern-Gemeinschaft entwickelt werden kann. ${ }^{10} \mathrm{Um}$ die angestrebte, agile „Veränderungsbereitschaft" in der Qualitätsarbeit des Akteurs Hochschule zu ermöglichen, wurde institutionelle Autonomie zur Ausgestaltung kontextuell passender Kulturen, Strukturen und Prozesse eingeräumt. Zeitgleich wurde der (legitime!) Anspruch auf Selbstverpflichtung und Transparenz, nach der Ausrichtung auf ein regelgeleitetes Ordnungssystem und der Nachweisführung effizienter und effektiver Leistungserbringung gestellt. ${ }^{11}$ Die österreichischen Fachhochschulen haben nicht zuletzt aufgrund der externen Qualitätssicherung ihre Qualitätsarbeit durch etablierte Modelle hoch professionalisiert und deren Güte mehrfach durch nationale wie internationale Auditierungen, Akkreditierungen und Zertifizierungen bewiesen. Demzufolge ist das Leistungsversprechen, das sich aus dem gesetzlichen Zielauftrag ableitet, nachhaltig gut und als positiv zu beschreiben. Aus Sicht einer vielfach evaluierten, auditierten und zertifizierten Hochschuleinrichtung wie der Fachhochschule Oberösterreich ist es herausfordernd, dem sehr hohen - und zwischenzeitlich auch sehr detaillierten - Nachweisumfang bei externen Qualitätskont-

$5 \quad$ Rekurriert auf das lateinische „valere“; stark, kräftig sein

6 Damals Kriterien, die innerhalb der European Association for Quality Assurance in Higher Education (ENQA) nationenübergreifend in Kooperation mit der European Students' Union (ESU), der European Association of Institutions in Higher Education (EU-RASHE) und der European University Association (EUA) entwickelt wurden.

$7 \quad$ Hier zu versehen als organisationale Rahmensetzungen, die Prozess-, Arbeits-/Ressourcen-, Produkt- und Dienstleistungsbereiche umschließen.

$8 \quad$ Nach dem lateinischen auditio: Anhörung.

9 Standards und Leitlinien für die Oualitätssicherung im europäischen Hochschulraum (ESG), Beiträge zur Hochschulpolitik, Nr. 3 (20159, Bonn, S. 12.)

10 Deci, E./Ryan, R. (1993): Die Selbstbestimmungstheorie der Motivation und ihre Bedeutung für die Pädagogik, Zeitschrift für Pädagogik, Jg. 39, Nr. 2, S. 223-238. Metzger, Ch. (2013): Lernhandeln und Lernmotivation. Überlegungen zum integrierten Lern- und Handlungsmodell in: Reinmann, G., et al. (Hrsg.): Hochschuldidaktik im Zeichen von Heterogenität und Vielfalt. Doppelfestschrift für P. Baumgartner und R. Schulmeister, Norderstedt, Books on Demand GmbH, S. 183-196

11 Berka, W. (2002): Autonomie im Bildungswesen. Zur Topographie eines bildungspolitischen Schlüsselbegriffs, Wien/Köln/Graz, Böhlau Verlag. Stensaker, B./ Harvey, L. (2011): Accountability in Higher Education. Global Perspectives on Trust and Power, New York, Routledge. 
rollen zu entsprechen. Dazu erwies sich das rasche Organisationswachstum, die Binnendifferenzierung der Leistungsbereiche in zwischenzeitlich fünf $\mathrm{GmbHs}$ in Form einer "Holding-Konstruktion"12 sowie die Dislozierung an vier Fakultäten in Hagenberg, Linz, Steyr und Wels als anspruchsvoll. Mit dem Ziel, ein leistungsfähiges, ganzheitliches System der breiten Teilhabe und Verantwortungsübernahme zu entwickeln und einzusetzen, wurde der methodische Zugang des EFOM-Modells gewählt. Dieser Zugang ermöglicht eine holistische Rahmensetzung und thematisiert die intern wie extern qualitätsrelevanten Leistungsbereiche im Sinne von sogenannten „Befähiger"-Dimensionen: Führung, Politik/Strategie sowie Mitarbeiter*innen und verbindet diese am Wege definierter, umzusetzender Prozesse mit der "Ergebnis"-Seite in Form von mitarbeiter-, kunden- und gesellschaftsbezogenen Mehrwert- und Nutzenmessungen/-darstellungen. Mittels dieses Zugangs werden auch Wechselwirkungen und Interdependenzen erkennbar und steuerbar. Der Qualitätsregelkreis wird entlang dieser Parameter durch regelmäßige interne wie externe Reflexion mit dem Ziel der Innovation und des Lernens auf Individuums- wie Organisationsseite geschlossen. Strukturell wurde ein alle Leistungsbereiche erfassendes, fakultäts- und kurienübergreifend zusammengesetztes Gremium eingerichtet ${ }^{13}$, dem ein ressortzuständiges Mitglied der Hochschulleitung vorsteht. Der Umstand, dass ein Mitglied des Gremiums auch Mitglied des Kollegiums ist, ermöglicht zudem unmittelbare Abstimmung und Kommunikation in der QS-Arbeit. Organisationsübergreifende Projekte, Initiativen, Vorträge, die Befragungen der Studierenden und weiterer Stakeholder sowie regelmäßige Berichte und Newsletter an die Hochschulleitung, das Kollegium sowie die Mitarbeitenden gewährleisten Einbindung und schaffen die Basis für Qualitätsbewusstsein und Verantwortungsübernahme. Mit Darstellung dieser Herangehensweise wird deutlich, dass zur Realisierung des gelingenden Zusammenspiels nicht nur einzelne „OM-Beauftragte", sei es auf Hochschulleitungs- oder auch Qualitätssicherungsebene mit entsprechender, qualitätsgeleiteter Leistungserstellung in Zusammenhang stehen, sondern sich ein auf Qualitätsaspekte und -kultur beruhender dialogbasierter Zugang entwickeln bzw. etablieren konnte. Dieser Ansatz ermöglichte und vereinfachte auch die mit 2012 gesetzlich eingeführte Verpflichtung zum Aufbau eines OM-Systems unter Berücksichtigung der "Zweiteilung“ in der Qualitätsverantwortung zwischen Erhalter und Kollegium. ${ }^{14}$ Die Verschränkung interner mit externer Qualitätssicherungsverfahren erfolgte in erster Linie durch Kopplung der OS-Prozesse sowie durch Abstimmung der jeweiligen Durchführungszeitpunkte/-phasen. Demzufolge setzt das OM-System der FH Oberösterreich auf ein alternierendes Wechselspiel zwischen Selbst- und Fremdreflexion sowohl auf individueller als auch auf organisationaler Ebene. Die daraus erzielten Ergebnisse und Erkenntnisse werden genützt, um die weiterführende Gestaltung und Veränderung von Qualitätskultur, Qualitätsstrategie und der Umsetzungsprozesse in Gang zu setzen.

\section{KRITISCHE REFLEXION DER QUALITÄTSARBEIT: FITNESS FOR (WHICH) PURPOSE?}

Vor dem Hintergrund der beschriebenen OM-Arbeit ist in der hochschulischen Praxis und in der Kombination aus interner und externer Qualitätssicherung der Aufbau einer "gemeinsamen Basis" bisweilen herausfordernd: Es gilt, unterschiedliche Interessen und Erwartungshaltungen, teils auch zusätzliche, „nicht-hochschulische“ legistische Grundlagen zu berücksichtigen. ${ }^{15}$ In diesem Zusammenhang ist auch zu fragen, wofür oder für wen hochschulische Qualitätssicherung einen Mehrwert darstellt? Denn es steht der Sektor und jede einzelne Fachhochschule unter einem stetig steigenden Leistungsdruck, zumal externe Qualitätssicherung nicht nur der Legitimation gilt, sondern das erfolgreiche „Bestehen" dieser Überprüfungen mit existentieller Absicherung verbunden ist. Nur wer in der Bewertung glänzt, kann mit Fortbestand, Wachstum und Reputation rechnen. Betrachtet man die Standards in Akkreditierungs- und Auditverfahren über den Zeitverlauf näher, so handelt es sich um einen zweifelsohne für die Stakeholder interessanten, wenngleich zunehmend umfänglicheren Nachweiskatalog in zahlreichen Dimensionen. Denn dieser umschließt nicht nur die Hinterfragung der Kernleistungsbereiche und -prozesse in Studium und Lehre sowie Forschung und Entwicklung, sondern umfasst auch die Nutzen- und Mehrwertgenerierung der hochschulischen Umwelt gegenüber. ${ }^{16}$ Zwischenzeitlich ist es herausfordernd zu entscheiden, welche noch weiteren Leistungen als "State-of-the-Art" zu erfüllen sind. Ist es das Personal und dessen Qualität? Sind es Anreizmodelle zur Leistungssteigerung? Sind es Maßnahmen für ökologische, ökonomische und soziale Nachhaltigkeit? Ist es der weitere (kostenintensive) Ausbau von Unterstützungs-, Servicierungsund Informationsangeboten? Ist es die Einführung weiterer Befragungen und Kennzahlen zur Leistungskontrolle? Sind es weitere Flexibilisierung und Durchlässigkeit und damit einhergehende organisatorische und strukturelle Diversifikation? Damit in Verbindung steht die Frage nach der Umfänglichkeit von Transparenz. Diese kann mittlerweile auch schon so manche Kommunikationsstrategie überfordern: Welche Informationen sollen für wen, in welchem Umfang, auf welcher Ebene der Homepage, in welchem zeitlichen Abstand bzw. welcher Dauer bereitgestellt werden? Nach dem "cui bono" gefragt, ist die Qualitätssicherung im österreichischen FH-Wesen international gewiss beispielgebend - es werden so gut wie alle Stakeholder umfassend informiert und eingebunden. Angesichts der Fülle an Studiengängen und deren Dokumentation ist jedoch zu fragen, welche Leser*innenschaft hier qualifiziert informiert werden und wie das erforderliche und wesentliche Kontextwissen dabei auch transportiert werden kann. Und so wertvoll Meinungspluarlismus

\footnotetext{
12 Vgl. https://www.fh-ooe.at/ueber-uns/organisation/, abgefragt am 19.11.2019

13 Qualitätsmanagement-Konferenz der FH OÖ: vgl. https://www.fh-0oe.at/ueber-uns/qualitaet/qm-konferenz/, abgefragt am 19.11.2019

14 Vgl. HS-QSG BGBI. I 74/2011 §22 und FHStG BGBI. 390/1993 idgF.

15 Die FH Oberösterreich unterliegt zB aufgrund der Rechtsform dem GmbH-G, arbeits- und sozialrechtlichen Gesetzen und Verordnungen etc.

$16 \quad$ Hier verstanden unter den hochschulischen Stakeholdern: Politik, Wirtschaft/Industrie, Sozial- und Gesundheitsbereich, Gesellschaft.
} 
auch im Sinne des Erkennens von „blinden Flecken” sein kann - bisweilen wird es für die einzelne Hochschule zum Balanceakt, zu ein und demselben Sachverhalt unterschiedliche Ansichten adäquat darzustellen. Mit Blick auf die ESG wäre im Kontext der Qualitätsüberprüfung daher kritisch zu reflektieren ${ }^{17}$, welche oder auch wieviel dieser Leistungen im Sinne von "Fitness for Purpose" durch Qualitätssicherung trainiert bzw. unter Beweis gestellt werden sollen. Die international wie national definierten Nachweispflichten sowohl im Bereich der Akkreditierung, als auch der institutionellen Audits weisen vielfach redundante Verfahrensinhalte auf. Vor diesem Hintergrund ist die Aufwand-Nutzen-Dimension für alle beteiligten Akteure zu hinterfragen. Es könnten in einzelnen Verfahrenstypen auch jeweils andere Dimensionen in das Blickfeld der Qualitätssicherung einfließen. Wenn z.B. ein etabliertes OM-System einer Fachhochschule durch Expert*innen überprüft und durch eine qualifizierte Qualitätssicherungsagentur zertifiziert ist, erscheint im Sinne einer Verfahrenseffizienz und -effektivität die wiederholte Überprüfung im Zuge von Akkreditierungsverfahren nicht zielführend. Bei der Akkreditierung wäre beispielsweise die Konzentration der wissenschaftlich und berufsfeldbezogen qualifizierten Expert*innen auf die „Herzstücke“ eines Studiengangsantrags wie das Curriculum, die Modulbeschreibungen und das angestrebte Qualifikations- und Tätigkeitsprofil von Interesse. Dahingehend wäre zu beleuchten, inwieweit Kriterien/Standards in den Verfahren zueinander abgestimmt bzw. sich wechselseitig ergänzend gestaltet werden könnten.

\section{CONCLUSIO: QUALITÄTSSICHERUNG IM ÖSTERREICHISCHEN FH- SEKTOR: CUI BONO?}

Qualitätsarbeit an (Fach-)Hochschulen fordert eine fortgeschrittene Form eines anspruchsvollen Organisationslernens: Es geht darum, Rahmenbedingungen zu etablieren, die sicherstellen, dass neben der reinen Korrektur von Abweichungen auch die Überprüfung zugrunde gelegter Werte und Normen erfolgt und darüber hinaus rasch und effektiv weitere Erkenntnisse oder auch Erwartungshaltungen nach zusätzlichen Leistungen in den hochschulischen Alltag einfließen. ${ }^{18}$ Die FH Oberösterreich und viele weitere (Fach-)Hochschulen nützen dazu ganzheitlich ausgerichtete OM-Modelle und -Systeme, die allesamt zertifiziert sind und die erforderliche Transparenz durch ein professionelles Berichtsund Qualitätswesen hergestellt haben. Das "Dazulernen“ am Wege der Qualitätssicherung ist über den Zeitverlauf nicht nur begrifflich und methodisch, sondern auch aus der internen wie systembezogenen Governance-Warte erkennbar - viele Maßnahmen wurden gesetzt, um Qualität im Sinne von Güte zu sichern und zu managen. Dennoch ist mit Blick auf die weitere Entwicklung zu hinterfragen, welches Ausmaß von „noch mehr" und "noch detailreicher" an Informationen und Nachweisen für noch mehr (?) Qualität zuträglich sind. In diesem Falle wäre nämlich auch die Begrifflichkeit der Qualitätssicherung in Frage zu stellen: Was wird "gesichert", wenn sich alles laufend verändert? Als gedankliches Experiment wäre vorstellbar, die Qualitätssicherung und ihre Verfahren einer Meta-Betrachtung zu unterziehen und zu versuchen, aus einem distanzierteren Blickwinkel - einer Vogelperspektive - möglicherweise besseren Überblick über das große Ganze und die tatsächlich relevanten Informationen über den Leistungszustand der Hochschulen bzw. Hochschul-Systeme zu erhalten. In diesem Sinne entsteht möglicherweise in der Hochschul-Governance auch ein Diskussionsprozess, der weg von der sehr detailgetreuen Bestandswahrung von definierten Standards hin zum „Entwickeln lassen“ der Hochschulen und deren qualitätsbezogener Profilbildung führt.

\section{QUELLEN}

Kozar, G, 1999: Hochschul-Evaluierung, in: Schriftenreihe des FHR, WUV-Verlag, Wien

Deci, E./Ryan, R. (1993): Die Selbstbestimmungstheorie der Motivation und ihre Bedeutung für die Pädagogik, Zeitschrift für Pädagogik, Jg. 39, Nr. 2, S. 223-238. Metzger, Ch. (2013): Lernhandeln und Lernmotivation. Überlegungen zum integrierten Lern- und Handlungsmodell in: Reinmann, G., et al. (Hrsg.): Hochschuldidaktik im Zeichen von Heterogenität und Vielfalt. Doppelfestschrift für P. Baumgartner und R. Schulmeister, Norderstedt, Books on Demand GmbH, S. 183-196

Berka, W. (2002): Autonomie im Bildungswesen. Zur Topographie eines bildungspolitischen Schlüsselbegriffs, Wien/Köln/Graz, Böhlau Verlag. Stensaker, B./Harvey, L. (2011): Accountability in Higher Education. Global Perspectives on Trust and Power, New York, Routledge.

Jarvis, D. (2014): Regulating higher education: Quality assurance and neo-liberal managerialism in higher education-A critical introduction, Policy and Society, Jg. 33, Nr. 3, S. 155-166.

Argyris, Ch. (1977): Double-loop Learning in Organizations, Harvard Business Review, Jg. 55, Nr. 5, S. 115-125, Geißler, H. (2000): Organisationspädagogik: Umrisse einer neuen Herausforderung, München, Vahlen Verlag, Seufert, S., et al. (2015): Steigerung der Lern- und Innovationsfähigkeit von Unternehmen und Organisationen in: Hoffmann, Christian Pieter, et al. (Hrsg.): Business Innovation: das St. Galler Modell, Wiesbaden, Springer Fachmedien, S. 283-311. 


\section{ZUR FACHHOCHSCHULE OBERÖSTERREICH:}

Die Fachhochschule Oberösterreich begeht im Jahr 2019 ihr 25 -jähriges Bestandsjubiläum. Mit dem akademischen Jahr 2018/19 werden 31 Bachelor- und 37 Masterstudiengänge mit insgesamt 5.735 Studierenden an den vier Fakultäten in Hagenberg, Linz, Steyr und Wels betrieben. ${ }^{19}$ Die FH Oberösterreich hat in ihrer Bestandsgeschichte neben rd. 100 Akkreditierungen und Re-Akkreditierungen von FH-Studiengängen ${ }^{20}$ zwischenzeitlich in den Jahren 2003, 2008 zwei institutionelle Evaluierungen sowie 2014 ein institutionelles Audit zur Zertifizierung des OMSystems gem. §22 HS-QSG durchlaufen.

\section{AUTORIN}

Prok. Dr. Regina Aichinger MSc, sozial- und wirtschaftswissenschaftliche Schwerpunkte. Doktorat im Bereich Betriebspädagogik. Mitglied der Hochschulleitung der FH Oberösterreich, Leitung Hochschulforschung und -entwicklung, Sprecherin des Netzwerks Hochschulforschung Österreich. Schwerpunkte: Strategische Positionierung und Hochschulforschung zu den Bereichen Governance, Organisationsentwicklung, Qualitätsmanagement und Diversity Management.

PROK. DR. REGINA AICHINGER MSC

Mitglied der Hochschulleitung der FH Oberösterreich

Franz-Fritsch-Straße 11, 4600 Wels

E: regina.aichinger@fh-ooe.at 